\title{
Quantum Cascade Surface-Emitting Photonic Crystal Laser
}

\section{Citation}

Colombelli, Raffaele, Kartik Srinivasan, Mariano Troccoli, Oskar Painter, Claire F. Gmachl, Donald M. Tennant, A. Michael Sergent, Deborah L. Sivco, Alfred Y. Cho, and Federico Capasso. 2003. “Quantum Cascade Surface-Emitting Photonic Crystal Laser." Science 302 (5649): 137477. https://doi.org/10.1126/science.1090561.

\section{Permanent link}

http://nrs.harvard.edu/urn-3:HUL.InstRepos:41371493

\section{Terms of Use}

This article was downloaded from Harvard University's DASH repository, and is made available under the terms and conditions applicable to Other Posted Material, as set forth at http:// nrs.harvard.edu/urn-3:HUL.InstRepos:dash.current.terms-of-use\#LAA

\section{Share Your Story}

The Harvard community has made this article openly available. Please share how this access benefits you. Submit a story.

Accessibility 


\section{Supporting Online Material Quantum Cascade Surface-Emitting Photonic-Crystal Laser}

Raffaele Colombelli, Kartik Srinivasan, Mariano Troccoli, Oskar Painter, Claire F. Gmachl, Donald M. Tennant, A. Michael Sergent, Deborah L. Sivco, Alfred Y. Cho, and Federico Capasso

In this section, we present supplementary information on the fabrication and numerical simulations of the PC QC lasers. More detailed expositions on these topics will be available in upcoming articles $(S 1, S 2)$.

$\underline{\text { Materials and Methods }}$

The processing steps are briefly reviewed here. After depositing a silicon dioxide mask layer ( 500 nm-thick), the PC pattern is written using electron beam lithography, transferred to the underlying mask via reactive ion etching, and then into the semiconductor using a chlorine-based inductively-coupled plasma reactive ion etch that produces nearly 90-degree sidewalls (Fig. 1B,D). An electrically-insulating silicon nitride $\left(\mathrm{Si}_{\mathrm{x}} \mathrm{N}_{\mathrm{y}}\right)$ layer is then deposited, and hexagonal windows are opened at the PC device sites. No mesa is etched around the devices, and thus only the openings in the $\mathrm{Si}_{\mathrm{x}} \mathrm{N}_{\mathrm{y}}$ define the regions of current injection. A low resistance $\mathrm{Ti} / \mathrm{Au}(30 / 300 \mathrm{~nm})$ contact is deposited on the $\mathrm{Si}_{\mathrm{x}} \mathrm{N}_{\mathrm{y}}$ to enable wire bonding (Fig. S1). The boundary of the insulating nitride layer is 5-10 $\mu \mathrm{m}$ from the edge of the etched PC, resulting in current injection into unpatterned 
regions. This inefficiency will be addressed in future works and is likely a key factor limiting the emission from highly localized defect states (see supporting online text). The sample is thinned, a metal back-contact $(\mathrm{Au} / \mathrm{Ge} / \mathrm{Ag} / \mathrm{Au})$ is deposited, and the surfaceplasmon-carrying metal layer $(\mathrm{Ti} / \mathrm{Au}, 10 / 100 \mathrm{~nm})$ is vertically evaporated onto the sample. The stringent requirement on the etched sidewall verticality is now evident: the topside contact must be evaporated while preventing electrical shorting within the etched holes.

\section{$\underline{\text { Design }}$}

An intuitive check of the photonic band-structure description given in the main text can be made by considering simple second-order Bragg diffraction. Second-order Bragg diffraction occurs when the in-plane wavevector $\left(\mathbf{k}_{\|}\right)$approaches that of a primitive reciprocal lattice vector $\left(\left|\mathbf{G}_{\mathrm{i}}\right|=4 \pi / \sqrt{3} \mathrm{a}\right)$ of the hexagonal lattice. Close to the second-order Bragg condition $\left(\left|\mathbf{k}_{\|}\right|=\left|\mathbf{G}_{i}\right|=4 \pi / \sqrt{ } 3 \mathrm{a}\right)$, coupling occurs to plane waves with near-zero inplane momentum, allowing light to radiate for surface emission. This is a consequence of the conservation of crystal-momentum in the scattering of plane-waves in such periodic structures, where wavevector (momentum) differences of a reciprocal lattice vector can be compensated for by the periodic polarization of the dielectric lattice $(S 3)$. Using the second-order Bragg-condition, the requisite lattice spacing for laser emission at $\lambda \approx 8 \mu \mathrm{m}$ can be estimated. From the filling fraction of dielectric material in the hexagonal lattice

$\left(f=1-(2 \pi / \sqrt{ } 3)(\mathrm{r} / \mathrm{a})^{2}\right)$ the average modal index is $n_{\text {avg }} \sim 1+\left(n_{\mathrm{eff}}-1\right) f=2.58$, which gives a second-order grating period $a \approx(2 \lambda) /\left(\sqrt{ } 3 n_{\text {avg }}\right) \approx 3.57 \mu \mathrm{m}$ at $\lambda=8 \mu \mathrm{m}$. This lattice constant value is in good correspondence with that obtained from the band-structure for 
the higher frequency $C$ region of Fig. 1A, indicating that the modes within the lower $A$ and $B$ frequency regions are more strongly tied to the high-dielectric semiconductor and should tune more slowly versus $r / a$, as confirmed by both numerical calculations and experimental measurements (Fig. 2C).

\section{Spatial distribution and polarization of the intensity patterns: mode assignment}

A two-dimensional array of PC QC microcavities is shown in Fig. S1A. The image was taken with a micro-bolometer camera sensitive in the wavelength range $7 \mu \mathrm{m}<\lambda<14 \mu \mathrm{m}$, while one device was being operated above laser threshold (see inset). An SEM image of a wire-bonded laser device is instead shown in Fig. S1B.

Fig. S2A-B shows two typical measured far-field intensity patterns of the lasing mode from PC microcavities with and without a central defect region, respectively. In these measurements the far-field emission pattern normal to the surface of the semiconductor chip was mapped out by scanning a $300 \mu \mathrm{m} \times 300 \mu \mathrm{m}$ nitrogen-cooled $\mathrm{HgCdTe}$ detector at a distance of approximately $10 \mathrm{~cm}$ without any intermediate optics. The average surface-normal far-field polarization of the lasing mode (Fig. S2D), measured by using a lens to focus the light on the $\mathrm{HgCdTe}$ detector with a polarizer placed in front of it, was found to be predominantly polarized along the $\left[{ }^{\wedge} \mathrm{y}\right]$-direction (cavity orientation as in Fig. 1B). In addition, as mentioned in the main text, we studied the polarized spatial distribution of the vertically emitted field intensity by placing a polarizer in front of a micro-bolometer camera fitted with a lens (Fig. 4). 
From the $\left[{ }^{\wedge} \mathrm{x}\right]-$ and $\left[{ }^{\wedge} \mathrm{y}\right]$-polarized intensity patterns (cavity orientation as in Fig. $1 \mathrm{~B}$ ), the symmetry of the lasing mode may be determined (S4-S6). For example, the nodal lines (lines of near-zero intensity in the images) along the $\left[{ }^{\wedge} \mathrm{x}\right]-$ and $\left[{ }^{\wedge} y\right]-a x i s$ of Fig. $4 B$ for the $\left[{ }^{\wedge} y\right]-$ polarized intensity pattern are consistent exclusively with an electromagnetic field mode which is odd (parity -1) under a mirror symmetry about the $\left[{ }^{\wedge} \mathrm{y}\right]$-axis and which is even (parity +1 ) under a mirror symmetry about the $\left[{ }^{\wedge} \mathrm{x}\right]$-axis. In the mathematical notation of group theory, such a mode is said to have $\mathrm{B}_{1}$ symmetry, where $\mathrm{B}_{1}$ is the label of one of the irreducible representations (IRREPS) of the point group of a rectangle $(S 7)$. A similar conclusion is reached by studying the $\left[{ }^{\wedge} \mathrm{x}\right]$-polarized intensity pattern of Fig. 4A which has anti-nodes on both the $\left[{ }^{\wedge} \mathrm{x}\right]-$ and $\left[{ }^{\wedge} \mathrm{y}\right]$-axis. Thus, the two polarized intensity patterns of Figs. 4A-B indicate that the laser emission is single mode and that the laser mode is of $\mathrm{B}_{1}$ symmetry.

To study the vertical emission characteristics of the modes of the PC microcavity, full three-dimensional (3D) FDTD simulations of the hexagonal symmetry non-defect cavity were performed. The etched hole depth was taken to be 5 microns and a $200 \mathrm{~nm}$ thick idealized "perfectly conducting" metal top contact was used to guide the TM surface wave (at a wavelength of $\approx 8 \mu \mathrm{m}$ this is a good approximation for a Au metal contact $(S 8, S 9))$. In order to reduce the size and time of the simulation, the cavity was limited to 6 periods of the hexagonal lattice (as opposed to the experimental 10 periods). Mirror boundary conditions were used to project the modes of the hexagonally symmetric cavity onto a basis compatible with the symmetry of a rectangle whose principal axes lie along the $\left[{ }^{\wedge} \mathrm{x}\right]-$ and $\left[{ }^{\wedge} \mathrm{y}\right]$-axis of the PC microcavity (save for numerical and discretization error, this does not reduce the symmetry of the non-degenerate modes of the hexagon). 
The highest frequency resonant mode of the A-peak was found to be of $\mathrm{B}_{1}$ symmetry (we refer to this mode simply as the $\mathrm{B}_{1}$ mode from here on). This is consistent with the experimentally measured emission spectrum (Fig. 2D) and with the laser near-field symmetry (Fig. 4A-B) as discussed above. Fig. S3A-B show the $\left[{ }^{\wedge} \mathrm{z}\right]-$ component (i.e. normal to the semiconductor-metal surface) of the electric field $\left(E_{z}\right)$ and its spatial Fourier transform $\left(\tilde{E}_{z}\right)$ for the $B_{1}$ mode. The values of the electric field have been sampled in the plane beneath the metal contact in the semiconductor active region. It is interesting to note, in hindsight, that this mode has only a small overlap with the central region of the PC cavity. This characteristic may partially explain the preferential selection of the $\mathrm{B}_{1}$ mode as the lasing mode. Due to the expected in-plane spreading resistance in the etched PC region, the current injection (and consequently the gain) is most likely higher in the periphery of the PC. Furthermore, the $\mathrm{B}_{1}$ mode's small overlap with the central region of the PC cavity is consistent with the similarity of the measured frequencies of the laser mode for both the defect and non-defect cavities. As a matter of fact, the entire electroluminescence spectra of both types of cavities are very similar. The noticeable absence - in cavities with a central defect- of the theoretically expected highlylocalized defect mode resonances between the A- and B-peaks also lends support to the theory of higher current injection in the periphery of the PC.

Calculations of the vertically emitted radiation field, taken in a plane several wavelengths above the PC cavity surface, were performed by eliminating the non-propagating FDTD near-field components and introducing - to simulate the experimental conditions - a $30^{\circ}$ collection angle cut-off for the imaging optics. The resulting $[\wedge x]-$ and $[\wedge y]-$ polarized intensity patterns of the $\mathrm{B}_{1}$ mode (shown in Figs. 4C-D) closely match the micro- 
bolometer camera measurements of Fig. 4A-B. The theoretical far-field emission pattern was also calculated by transforming the FDTD generated radiation field into the far-field (S10) and it is shown in Fig. S2C. The increased intensity of the two lobes on the [^y]axis in Fig. 4A of the $\left[{ }^{\wedge} \mathrm{x}\right]$-polarized intensity and in Fig. S2C of the far-field pattern are a result of inadvertent symmetry breaking of the hexagonal symmetry of the PC cavity due to discretization error in employing the mirror boundary conditions in the FDTD simulation. In this case, the computed near-field under the metal contact is still very symmetric, as shown in Fig. S3A. As discussed in footnote $S 7$, the symmetry breaking in the measured far-field (experimentally we observe the opposite effect, i.e. more intensity in the $[\wedge$ y]-polarization (Fig. 4B, Fig. S2D), is most likely a result of a similar subtle near-field effect due to the rectangular metal contact geometry.

The 3D FDTD calculated radiative quality-factor $(\mathrm{Q})$ of the $\mathrm{B}_{1}$ mode was found to be roughly a factor of two higher than the $\mathrm{Q}$ value of the neighboring A-peak resonant modes, helping explain why it is the first mode to lase. The calculated in-plane, top (vertical), and bottom (substrate) radiation rates, as given by effective cavity qualityfactors, were $\mathrm{Q}_{\|}=600, \mathrm{Q}_{\mathrm{v}}=10,000$, and $\mathrm{Q}_{\mathrm{s}}=7,000$, respectively. An estimate for the $\mathrm{Q}$ value associated with absorptive loss in the metal and semiconductor at 8 microns $(\alpha=$ $\left.40 \mathrm{~cm}^{-1}\right)$ is, $\mathrm{Q}_{\mathrm{a}}=800$. Thus, the vertical extraction efficiency of the PC microcavity laser is estimated to be, $\eta_{v}=Q_{v}^{-1} /\left(Q_{\|}^{-1}+Q_{v}^{-1}+Q_{s}^{-1}+Q_{a}^{-1}\right)=6 \%$. The vertical extraction of light in this case is due to the fact that the resonances forming the A-peak sit near the $\Gamma$ point of the reciprocal lattice (Figs. 1A, S3B). They therefore contain small in-plane Fourier components which can radiate into the surrounding air, exactly as in a second order distributed Bragg reflector. The coupling of radiation from the bottom 
semiconductor-metal interface to the top metal-air interface (from which the radiation finally escapes) is mediated through the air holes, as well as through the metal itself (although this effect has not been included in our calculations) due to the fact that the metal contact layer in the PC region is only $100 \mathrm{~nm}$ thick, slightly below the skin depth at 8 micron in $\mathrm{Au}(\sim 150 \mathrm{~nm})(S 8)$. 


\section{References and Notes}

S1. R. Colombelli et al., manuscript in preparation (unpublished).

S2. K. Srinivasan et al., manuscript in preparation (unpublished).

S3. A. Yariv, Optical Electronics, 4th ed., Saunder College Publishing, a division of Holt, Rinehart and Winston, Inc., Orlando, Florida, 1991.

S4. J. D. Joannopoulos, R. D. Meade, and J. N. Winn, Photonic Crystals, Princeton University Press, Princeton, New Jersey, 1995.

S5. M. Tinkham, Group Theory and Quantum Mechanics, International Series in Pure and Applied Physics, McGaw-Hill, Inc., New York, NY, 1964.

S6. O. Painter and K. Srinivasan, Phys. Rev. B. 68, 035110 (2003).

S7. One may use group theory ( $S 6$ ) to categorize the eigenmodes of a given electromagnetic system according to the its spatial symmetries $(S 4, S 5)$. In the case of a hexagonally symmetric non-defect cavity, one must consider the $\mathrm{C}_{6 \mathrm{v}}$ symmetry point group, which supports a non-degenerate $\mathrm{B}_{1}{ }^{\prime}$ IRREP (there is also the possibility that the laser mode is coming from a degenerate E1 IRREP space, although FDTD simulations show this is not the case, as discussed in the text) which shares all the same transformation properties of the $\mathrm{B}_{1}$ IRREP of $\mathrm{C}_{2 \mathrm{v}}$ under the symmetries of a rectangle. Additionally, the $\mathrm{B}_{1}{ }^{\prime}$ IRREP transforms into itself under the remaining symmetries of a hexagon (six- and three-fold rotations, and corresponding rotated mirror planes). Thus, for a perfectly hexagonal cavity, the near-field and far-field of the non-degenerate $\mathrm{B}_{1}{ }^{\prime}$ mode should have six-fold rotation symmetry. For both the PC microcavities with and without a central defect region we measure similar far-field intensity patterns for the band-edge laser mode (Fig. S2A-B). These intensity patterns, however, do not have the full symmetry of the hexagon. This is to be expected for the PC microcavity with a central defect as the defect reduces the symmetry of the cavity to that of $\mathrm{C}_{2 \mathrm{v}}$. The breaking of the six-fold symmetry in the experimentally measured far-field of the non-defect lasing mode must then be due to other, more subtle features of the PC microcavity lasers. The most likely candidate for this symmetry breaking is the rectangular shape of the outer metal contact pad used to inject current into PC. The rectangular geometry of the contact pad (that - in principle - can be probed by the surface plasmon waves on the top surface of the metallic contact pads) and/or the gain-guiding effects due to non-uniform current injection in the PC region can induce slight distortions in the near-field of the laser mode. In turn, the far-field radiation pattern is extremely sensitive to slowly varying field variations in the plane of the near-field (it is the small in-plane Fourier components of the field which are radiated into the air above the cavity). 
S8. $\quad$ L. Martin-Moreno et al., Phys. Rev. Lett. 86, 1114 (2001).

S9. J. D. Jackson, Classical Electrodynamics, 2nd ed., John Wiley \& Sons, Inc., New York, NY, 1975.

S10. J. B. Judkins and R. W. Ziolkowski, J. Opt. Soc. Am. B 12, 1974 (1995). 

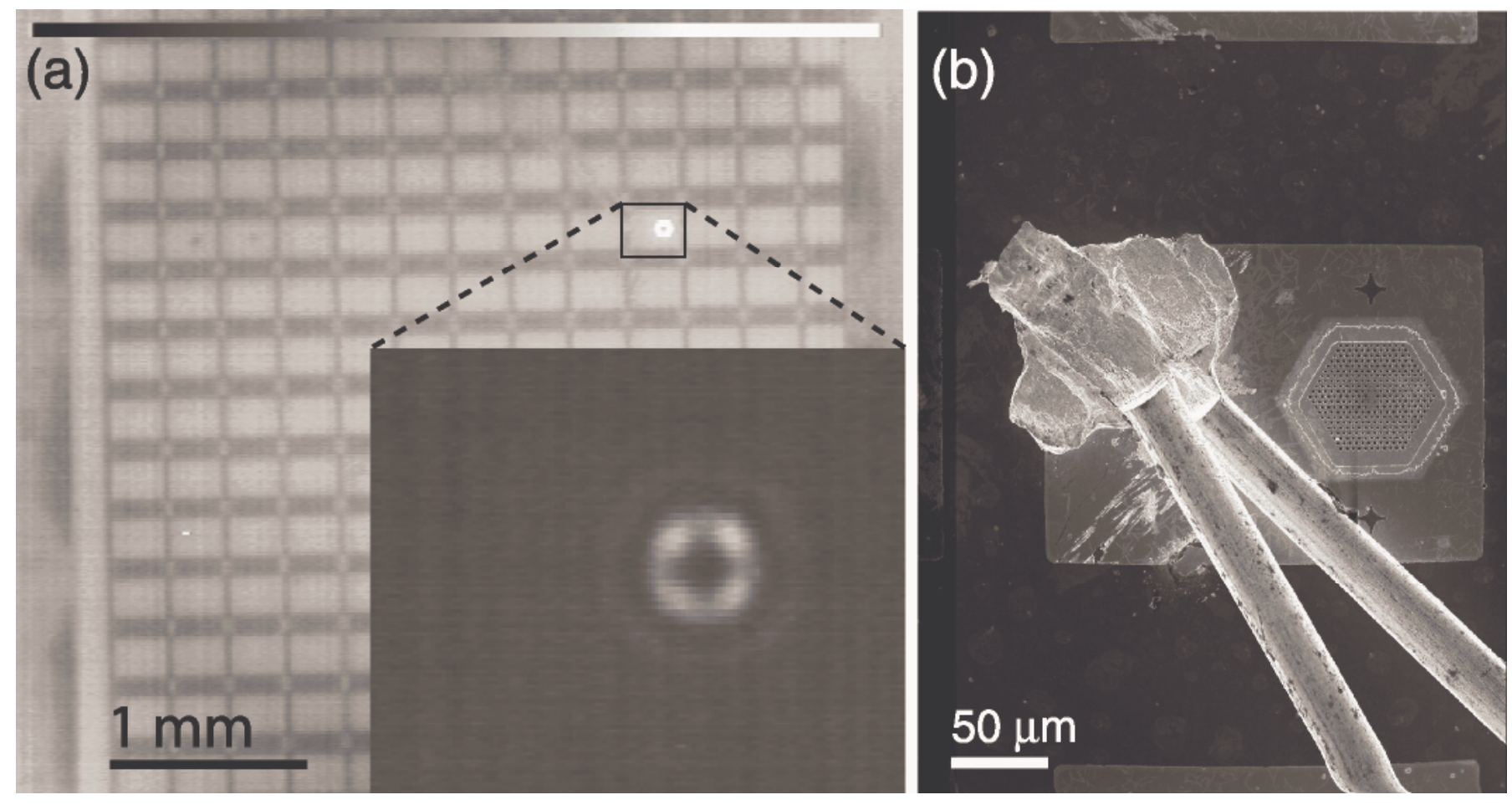

Figure S1: (A) Micro-bolometer camera image of a 2D array of PC QC lasers. Each rectangle corresponds to the outer metal top contact of a laser device. The microbolometer camera is sensitive in the range $7 \mu \mathrm{m}<\lambda<14 \mu \mathrm{m}$. The inset shows a close up of the lasing device emission, while the laser was being operated in pulsed mode at 100 $\mathrm{kHz}$ repetition rate with a pulse width of $40 \mathrm{~ns}$. Since several other devices were functional on this same chip (they were not operated simultaneously, though) the image indeed shows an array of single mode, lithographically tunable, vertically emitting QC lasers. (B) SEM image of a wire-bonded laser device. The central patterned region sits in a hexagonal opening in the rectangular metal contact pad. The total device size of each element in the $2 \mathrm{D}$ array is $210 \times 150 \mu \mathrm{m}$, with the PC cavity region roughly $60 \mu \mathrm{m}$ in diameter. 

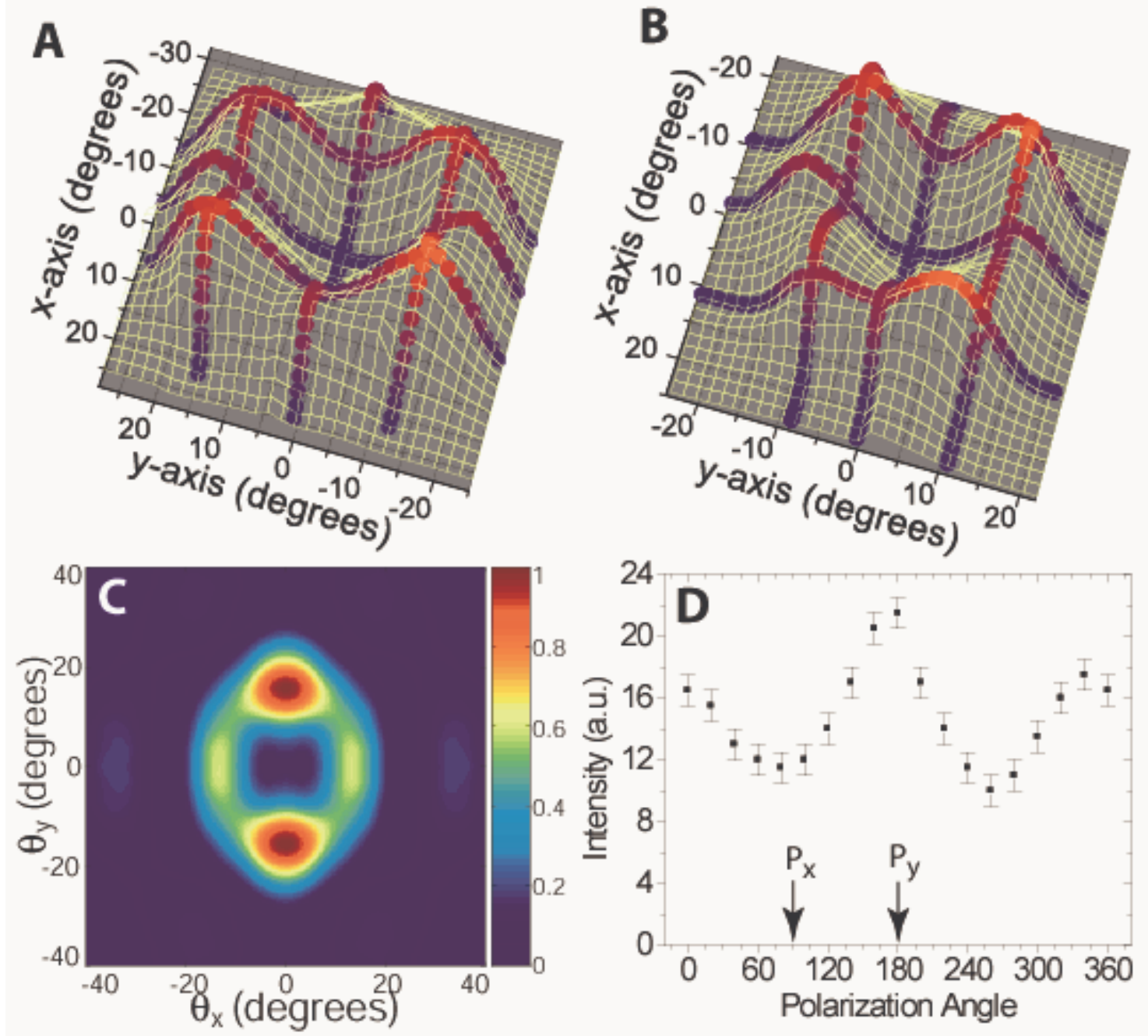

Figure S2: Far-field emission pattern of the PC microcavity lasers (A) with and (B) without central defect regions. The far-field pattern of a laser structure with a central defect region shows a slightly more elongated shape along the $\left[{ }^{\wedge} \mathrm{y}\right]-$ axis of the PC cavity (the extended direction of the central defect region - cavity orientation as in Fig. 1B). The measurements were performed by scanning a nitrogen-cooled $\mathrm{HgCdTe}$ detector in front of the laser device with no intermediate optics. The experimental data are shown as blue/red dots. An interpolation of the line scans (shown as a light yellow grid) is used to generate an approximate 2D intensity image. (C) FDTD simulation of the far-field intensity pattern for the high-frequency mode within the A-peak set of resonances. The device simulated has no central defect and contains only 6 periods of the PC lattice. (D) Polarization dependence of the laser emission in the far-field. 

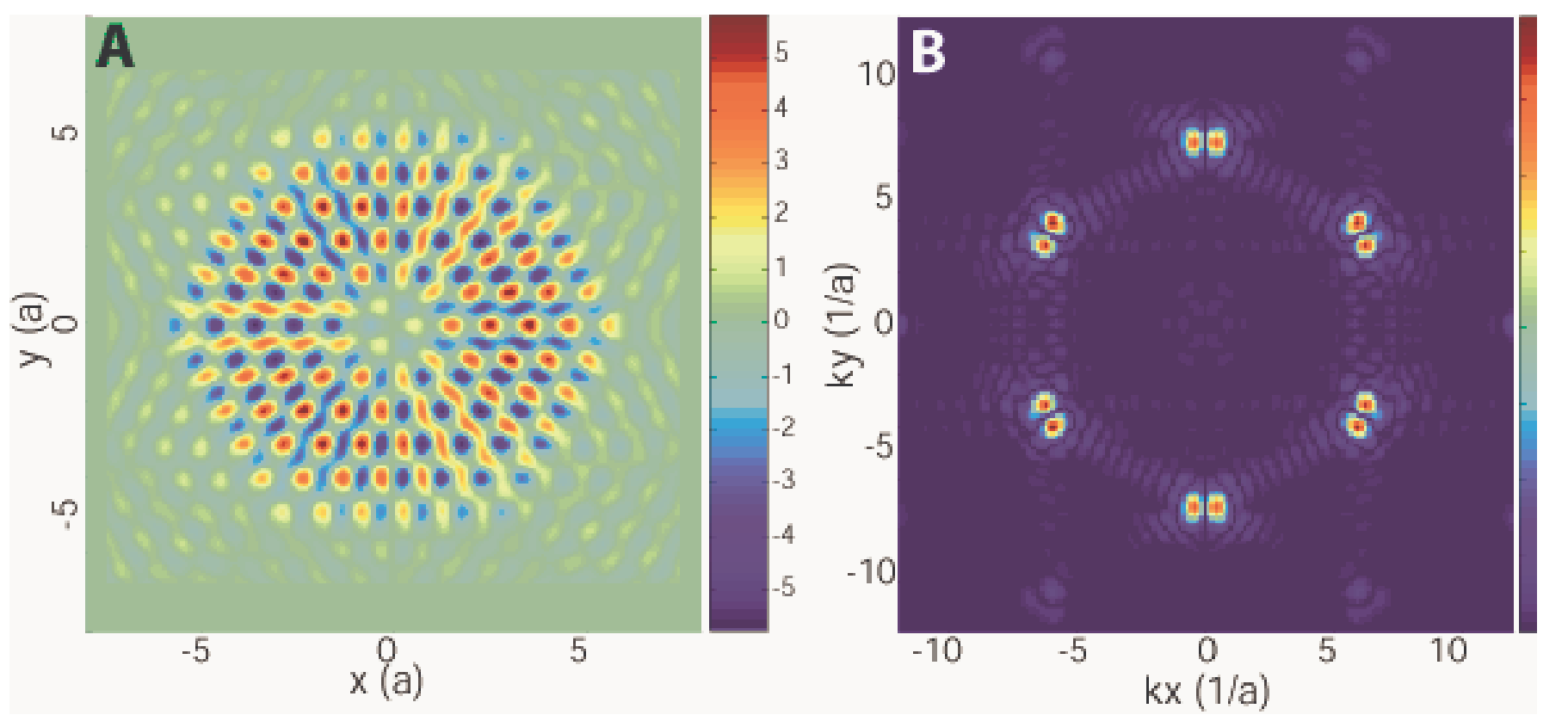

Figure S3: FDTD simulation of the high-frequency $\mathrm{B}_{1}$ symmetry mode lying within the A-peak frequency range. (A) Plot of $\mathrm{E}_{\mathrm{z}}$ (electric field normal to the semiconductor surface) in the plane of the PC cavity just beneath the metal contact in the semiconductor active region. (B) In-plane spatial Fourier transform of $\mathrm{E}_{\mathrm{z}}$, showing that the dominant Fourier components of the $\mathrm{B}_{1}$ symmetry mode come from the $\Gamma$-point in the reciprocal lattice. 